\title{
Protective Films on Metals
}

\begin{abstract}
$\mathrm{T}$ WO papers presented at the Autumn Meeting of the Institute of Metals by Drs. L. E. Price and G. J. Thomas dealing respectively with "The Tarnishing of Silver and Silver Alloys and its Prevention" and "Oxidation Resistance in Copper Alloys" may well represent the commencement of a new era in the investigation of alloy systems resistant to oxidation and scaling. It has been suggested by Wagner that for a film to provide marked resistance to further oxidation high electrical resistance, such as is offered by films of the pure oxides of aluminium, beryllium or silicon is required. This hypothesis is abundantly confirmed by the present authors' work.

When, however, alloys of silver or copper are heated in air under normal conditions, such resistant films cannot be produced owing to the fact that both the constituents of the alloy oxidize simultaneously, yielding a coat, the electrical resistance of which is not high. Optimum results can only be expected when films of a suitable pure oxide are formed. The problem, therefore, with which the authors were faced was the production of films of this type, and their solution of the difficulty calls for the highest praise. By an appropriate pre-treatment protective films of alumina have been formed on alloys of silver or copper con-
\end{abstract}

taining 1 per cent of aluminium, and remarkable resistance to oxidation and tarnishing both at room and at elevated temperatures has been obtained.

Two methods have been developed for the formation of such films of alumina or beryllia ; first by a selective oxidation of the aluminium or beryllium in the alloy by heating in hydrogen containing a partial pressure of water vapour of $0.1 \mathrm{~mm}$., or by the cathodic deposition of films of these oxides. Some idea of the magnitude of this research even in its present stage will be gathered from the fact that 70,000 specimens of silver and its alloys have been exposed for periods up to one year in fifteen different locations.

This work is as yet in its experimental stage, and until data are available regarding the mechanical properties of the films, and particularly their resistance to abrasion, it is not easy to visualize the practical possibilities of the process. It is probably true to say, however, that no more serious disservice to such practical application could be done than by inducing the authors prematurely to abandon their present purely scientific treatment of the subject and to devote their attention to the empirical and routine examination of alloys of possible industrial significance. F. C. T.

\section{Research on Concrete}

$\mathrm{T}$ HE steadily increasing use of concrete in the construction of buildings and roads has given added impulse to the efforts being made to ascertain how its quality may be improved and how it can be used to the best advantage. One of the most important of the factors which influence the properties of this material is the grading of the aggregate and new and valuable light has been thrown on this by the investigations made at the Building Research Station and at the Road Research Laboratory, the results of which have now been published*.

That the strength of concrete depends directly upon the ratio of water to cement and that other factors are of importance mainly in so far as they affect this ratio is now well established. It follows that the grading of the aggregate determines the strength of the concrete inasmuch as it influences the water content required to bring it to the desired degree of workability. The now widely known method of consolidating concrete by vibration may be used to obtain increased strength because it is possible thereby to consolidate a drier mix, thus reducing the necessary water: cement ratio. The term 'workability' has generally been held to convey an impression of the ease or otherwise with which a mix can be placed, but no precise definition has previously been attempted. It is shown here that the only part of the applied work which is usefully employed in the preparation of the mix is that used in overcoming the internal friction of the concrete and the friction between concrete and mould or reinforcement. Of this useful work, the only portion

* Department of Scientific and Industrial Research and Ministry of Transport, Road Research, Technical Paper No. 5. "The Grading of Aggregates and Workability of Concrete." (Iondon: H.M. Stationery Office.) $18.3 d$. depending on concrete alone is due to the internal friction and, from this consideration, workability is defined as the property which determines the amount of internal work required to compact the concrete.

For the measurement of this property, the slump test is shown to be defective in two respects, and the compacting factor test, finally chosen as suitable, is based on the above definition and measures the amount of compaction produced by the application of a standard quantity of work. In order to determine the effect of compactness a series of tests was carried out in which the specimens received different amounts of tamping and therefore contained different proportions of air-voids. The loss of strength due to a degree of under-compaction, as measured by the proportion of air-voids, was found to be 30 per cent when the voids were 5 per cent and as much as 90 per cent with 25 per cent of air-voids. It was shown also that, provided the specimens were fully compacted and had the same water-cement ratio, quite large variations of aggregate grading and shape of particles had no appreciable effect on strength. The grading is of importance only in so far as it affects workability and the water-cement ratio necessary to obtain the required workability, and its suitability should be judged in relation to the cement content and the degree of workability required.

The paper fully describes the tests made, and gives photographs in illustration of them, while the quantitative results are adequately set out in tables and curves. From the various data supplied it should be possible for the engineer to obtain the best combination of workability and strength for any particular purpose. 